\title{
Quantum dots shed light on processes in living cells
}

\author{
Donna J. Arndt-Jovin, Diane S. Lidke, Keith A. Lidke, Bernd \\ Rieger, and Thomas M. Jovin
}

New reagents and techniques in cell biology make it possible to visualize cell-signaling mechanisms in real time.

Water-soluble, biofunctionalized, semiconductor quantum dots (QDs) are fluorescent nanoparticles that have the advantage of much greater photostability compared with conventional fluorescent dyes. As a consequence, single QDs can easily be detected in living cells, and their localization can be monitored over minutes to days. ${ }^{1-4}$ We have shown that QDs coupled to growth-factor ligands behave similarly to unmodified growth factors, and serve as highly fluorescent probes of the erbB family of tyrosine kinase growth factor receptors. ${ }^{1}$

Single epidermal growth factor (EGF) peptides attached to QDs were used to activate epidermal growth factor receptors (EGFR, erbB1) by binding to the receptors. This caused the to form pairs (dimers) that, in turn, induced the uptake of the resulting QD-EGFR complex into internal vesicles, or endosomes. ${ }^{1}$ Continuous confocal laser scanning microscopy and flow cytometry measurements of QDs, combined with fusing visible fluorescent proteins to the receptors, allowed us to visualize individual steps in the signaling cascades initiated by these receptors in living cells (see Figure 1).

This approach has led to the exciting new discovery of a novel retrograde transport mechanism of the activated receptors on filopodia. ${ }^{1-3}$ The cytoskeleton of mammalian cells comprises a dynamic network of polymerized actin and tubulin, and their associated proteins, that mediates protein trafficking, cell motility, and endocytosis. The filopodia are fine sensory processes that extend from the cell body. They have a core of F-actin bundles that undergo growth and exchange by the addition of actin monomers to one end, at the tips of the filopedia, and depolymerization from the other, a process referred to as treadmilling. ${ }^{5}$ The process also involves active transport of the actin filaments by myosin molecular motors, culminating in a net flow of Factin directed toward the interior of the cell. Association of other macromolecules with an actin subunit within the filament of a
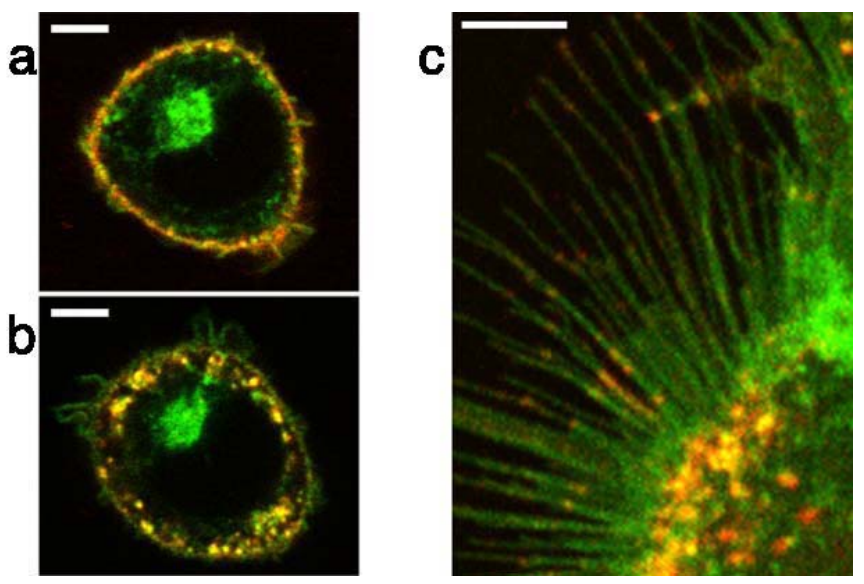

Figure 1. A QD-EGF binds and activates cells expressing a green fluorescent protein (GFP)-fused EGFR. (a) Red QD-EGF and green GFP-EGFR colocalize on the cell membrane within minutes and (b) are rapidly internalized together into endosomes in the cytoplasm. (c) Individual receptors on long sensory filaments called filopodia can be visualized by $Q D$-EGF binding. Images are single confocal sections through living cells.
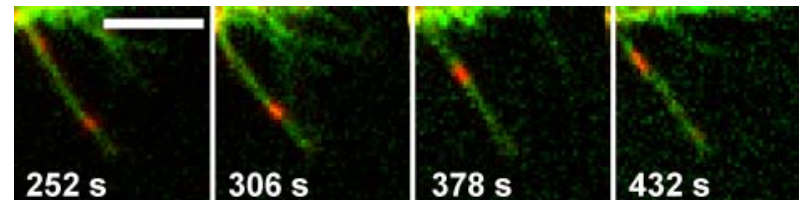

Figure 2. Snapshots in time showing retrograde transport of $Q D-E G F-$ receptor-activated complexes on a filopodium. ${ }^{1,2}$

filopodium causes that macromolecule to move toward the cell body in a process known as retrograde transport.

Using inhibitors of EGFR kinase as well as cytochalasin (a drug that depolymerizes actin), we showed that retrograde transport of the QD-EGFR complex occurs only after EGFloaded receptors dimerize and couple to the actin treadmill.

Continued on next page 


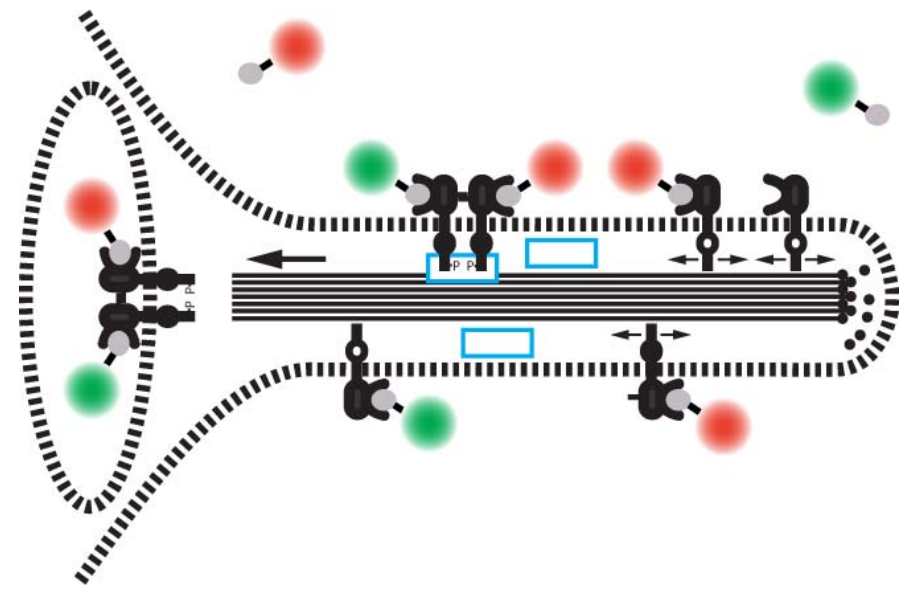

Figure 3. The scheme depicts the mechanism of filopodial retrograde transport. QD-EGF binds to EGFR monomers diffusing in the membrane. Dimerization of two liganded receptors activates the receptor tyrosine kinase, leading to autophosphorylation of the EGFRs and their attachment to the actin filament bundle in the filopodium through an adaptor protein complex denoted by the blue box. The QD-EGFR complex stops diffusing and is transported by actin treadmilling toward the cell body, into which it endocytoses through a clathrin-coated pit. ${ }^{2}$

Moreover, the receptors 'surf' on the external surface of the filopodia and are only endocytosed at their base after retrograde transport (Figure 2). ${ }^{2}$ These results suggest that the receptors situated on the filopodia play an important role as a sensory system (natural 'biosensors') for the organism. Such a system would assist in regulating signal transduction pathways through transport of receptors only after a given threshold of ligand binding is attained (see the model shown in Figure 3).
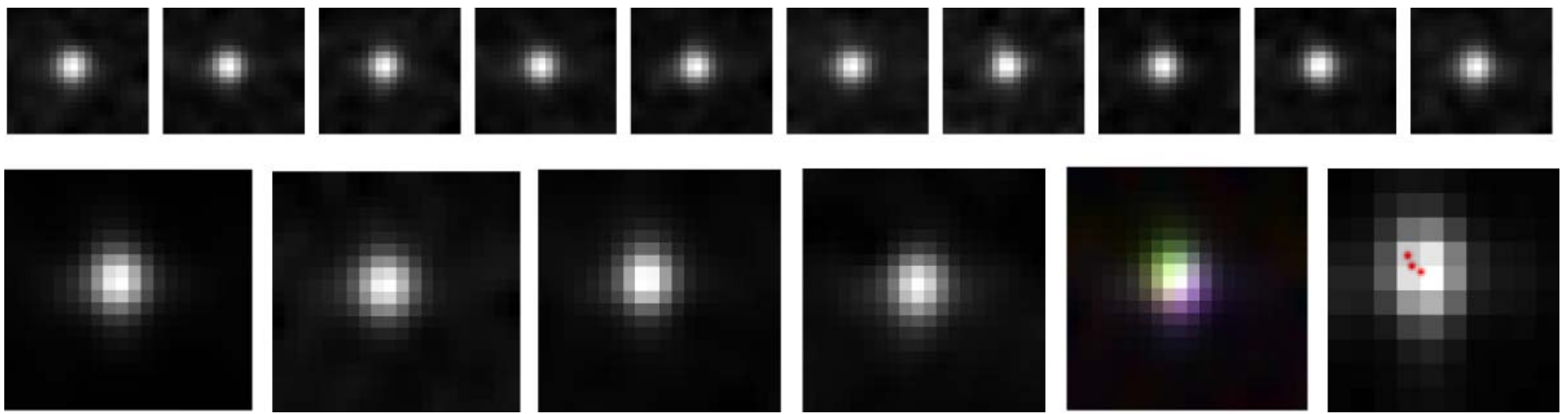

Figure 5. Independent component analysis (ICA) decomposition of blinking $Q D$ data sets showing the resolution of three $Q D$ s in one locus. The upper panel shows 10 frames from a time series. The lower panel shows left to right: time average of the series; ICA-returned component 1 ; ICA component 2; ICA component 3; color-coded overlay of components 1, 2, and 3; position overlay of 1,2 , and $3 .^{6}$ 
electron-hole pair, which on recombination results in emission of a less-energetic photon. The emission wavelength depends on the size of the core (smaller produces lower wavelength). Although the blinking behavior of QDs adds complexity to some live cell- imaging applications, ${ }^{3}$ it can be used to achieve superresolution in the light microscope ${ }^{6}$ (Figure 5). A more serious problem is that posed by QDs emitting >600nm: greater size owing to outer shells for passivation and for biocompatibility limit the ability of the QDs to form tight cell junctions. ${ }^{3}$ Noble metal nanodots constitute an attractive alternative to QDs in that they are much smaller and nontoxic. ${ }^{7,8}$ We have developed a new electrochemical method for synthesizing gold nanodots that allows us to reproducibly control cluster size. ${ }^{3}$

The in-vivo measurements using QDs described above provide new insights into processes and interactions that previously could only be studied on fixed cells or by biochemical fractionation. ${ }^{9}$ The combination of different QDs and even smaller fluorescent noble metal nanodots coupled to cellular constituents promises single-molecule resolution of the locus, aggregation state, and dynamics of those constituents in live cells.

The authors wish to thank the Max Planck Society and the EU for generous support.

\section{Author Information}

\section{Donna J. Arndt-Jovin}

Molecular Biology

Max Planck Institute for Biophysical Chemistry

Göttingen, Germany

http:/ / www.mpibpc.mpg.de/groups/jovin/

people/donna/index.html

http:/ / www.mpibpc.mpg.de/groups/jovin/

Donna Arndt-Jovin is head of the cell biology group at the MPI and past president of the International Society for Analytical Cytology. She has made seminal contributions to techniques for digital imaging, including Förster resonance energy transfer (FRET), fluorescent lifetime imaging microscopy (FLIM), and confocal microscopy. She has also presented papers at a number of SPIE BIOS conferences.

\section{Diane S. Lidke}

Dept. of Molecular Biology

Max Planck Institute for Biophysical Chemistry

Göttingen, Germany

Dept. of Pathology

University of New Mexico

Albuquerque, NM
Keith A. Lidke

Dept. of Molecular Biology

Max Planck Institute for Biophysical Chemistry

Göttingen, Germany

Sandia Laboratories

Albuquerque, NM

\section{Bernd Rieger}

Dept. of Molecular Biology

Max Planck Institute for Biophysical Chemistry

Göttingen, Germany

FEI Electron Optics

Eindhoven, The Netherlands

\section{Thomas M. Jovin}

Dept. of Molecular Biology

Max Planck Institute for Biophysical Chemistry

Göttingen, Germany

Thomas M. Jovin is an authority on FRET and FLIM and has developed a number of instruments based on fluorescence detection. He chaired the SPIE BIOS session on colloidal quantum dots in 2006.

\section{References}

1. D. S. Lidke, P. Nagy, R. Heintzmann, D. J. Arndt-Jovin, J. N. Post, H. E. Grecco, E. A. Jares-Erijman, and T. M. Jovin, Quantum dot ligands provide new insights into erbB/HER receptor-mediated signal transduction, Nat. Biotechnol. 22, pp. 198-203, 2004. doi:10.1038/nbt932

2. D. S. Lidke, K. A. Lidke, B. Rieger, T. M. Jovin, and D. J. Arndt-Jovin, Reaching out for signals: filopodia act as sensory organs via retrograde transport of activated EGF receptors, J. Cell Biol. 170, pp. 619-626, 2005. doi:10.1083/jcb200503140

3. D. J. Arndt-Jovin, M. A. Lopez-Quintela, D. S. Lidke, M. J. Rodríguez, F. M. Santos, K. A. Lidke, G. M. Hagen, and T. M. Jovin, In vivo cell imaging with semiconductor quantum dots and noble-metal nanodots, Proc. SPIE 6096, p. 60960. doi: $10.1117 / 12.646794$

4. T. M. Jovin, Quantum dots finally come of age, Nat. Biotechnol. 21, pp. 32-33, 2003. doi:10.1038/nbt 0103-32

5. J. V. Small, T. Stradal, E. Vignal, and K. Rottner, The lamellipodium: where motility begins, Trends Cell Biol. 12, pp. 112-20,2002. doi:10.1016/S0962-8924(01)02237-1

6. K. A. Lidke, B. Rieger, T. M. Jovin, and R. Heintzmann, Superresolution by localization of quantum dots using blinking statistics, Opt. Express. 13 (18), pp. 7052-7062, 2005. http:/ / www.opticsinfobase.org/abstract.cfm?URI=oe-13-18-7052

7. G. Woehrle and J. Hutchison, Thio-functionalized undecagold clusters by ligand exchange: synthesis, mechanism, and properties, Inorg. Chem. 44, pp. 6149-58, 2005. doi:10.1021/ic048686+S0020-1669(04)08686-0

8. J. Zheng, C. Zhang, and R. Dickson, Highly fluorescent, water-soluble, size-tunable gold quantum dots, Phys. Rev. Lett. 93, p. e077402, 2004. doi:10.1103/PhysRevLett.93.077402

9. G. Gur and Y. Yarden, Enlightened receptor dynamics, Nat. Biotechnol. 22, pp. 169170, 2004. doi:10.1038/nbt0204-167 